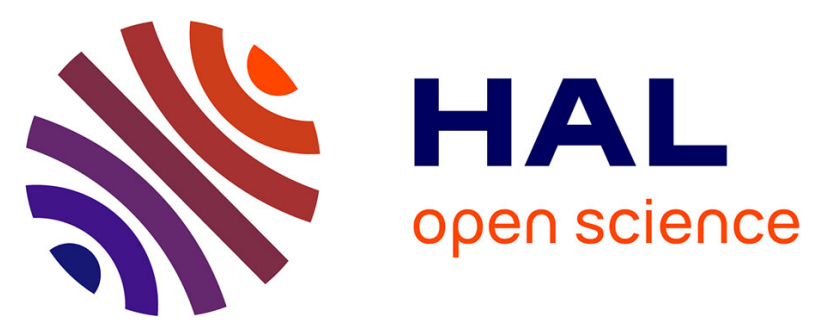

\title{
An efficient one-step-ahead optimal control for urban signalized traffic networks based on an averaged Cell-Transmission model
}

\author{
Pietro Grandinetti, Carlos Canudas de Wit, Federica Garin
}

\section{To cite this version:}

Pietro Grandinetti, Carlos Canudas de Wit, Federica Garin. An efficient one-step-ahead optimal control for urban signalized traffic networks based on an averaged Cell-Transmission model. ECC 2015 - 14th European Control Conference, Jul 2015, Linz, Austria. hal-01188535

\section{HAL Id: hal-01188535 \\ https://hal.science/hal-01188535}

Submitted on 31 Aug 2015

HAL is a multi-disciplinary open access archive for the deposit and dissemination of scientific research documents, whether they are published or not. The documents may come from teaching and research institutions in France or abroad, or from public or private research centers.
L'archive ouverte pluridisciplinaire HAL, est destinée au dépôt et à la diffusion de documents scientifiques de niveau recherche, publiés ou non, émanant des établissements d'enseignement et de recherche français ou étrangers, des laboratoires publics ou privés. 


\title{
An efficient one-step-ahead optimal control for urban signalized traffic networks based on an averaged Cell-Transmission model
}

\author{
Pietro Grandinetti, Carlos Canudas de Wit and Federica Garin
}

\begin{abstract}
This paper presents a model for large urban traffic networks, based on the well-known macroscopic Cell Transmission Model. We start by describing the dynamics of traffic flow at signalized intersections. Then we develop an average-based approximation of such a system, that we use to build our control algorithm as a linear optimization problem. Simulation results validate the averaged approach and show the effectiveness of the proposed control strategy.
\end{abstract}

\section{INTRODUCTION}

Traffic congestion on urban roads is a problem of great interest nowadays since it strongly affects security and pollution; effective and easy-to-handle models are therefore needed to represent and control traffic behavior. The scientific community relies on macroscopic models of time-space evolution of the traffic. Such models describe traffic as a fluid, and are based on a mass conservation law [1].

With respect to microscopic models, macroscopic ones are preferred due to their simplicity in characterizing vehicles' flows and densities. The Cell Transmission Model (CTM) [2] is an example, widely used, of this kind of representation.

In the last decades the major interest has been in analyzing and controlling highway traffic, and only recently the attention is moving on urban networks. The CTM's extension to networks was proposed in [3], and in [4] it has been shown the existence of a macroscopic fundamental relation between flow and density even for urban roads, with experimental validation.

In $[5,6]$ outflow is defined independently from downstream supply and it is then equal to a link's demand. This is unrealistic for traffic evolution, as pointed out in [7], since in such a way upstream flow is not affected by downstream congestion. Solutions to this problem are proposed in [7, 8].

Our contribution regarding traffic modelling consists first in the formulation of the traffic's dynamics as an extended CTM with FIFO policy at intersections; based on this, we then define an approximated averaged model, provide numerical validation of its quality, and use it as a tool to design traffic lights' control. Although the idea of representing traffic lights as the percentage of their green time (duty cycle) has been already employed in scientific works (e.g., [8, 9, 10]), authors employing this technique often base all their

This work has been partly funded by the EU FP7 project SPEEDD (619435).

Pietro Grandinetti is with the NeCS team and GIPSA-Lab, pietro.grandinettiegipsa-lab.fr

Carlos Canudas de Wit is Director of Research at CNRS, France, carlos.canudas-de-witegipsa-lab.fr

Federica Garin is Researcher with the NeCS team at Inria Rhône-Alpes, federica.garineinria.fr work on this approximated model, while we employ the averaged model as intermediate tool to design the control actions, which will then be actuated in the non-averaged network.

Our choice of traffic lights as control devices is motivated by a practical consideration: they are nowadays the main reason of more or less efficient network operations, even though they were originally installed only in order to guarantee the safe crossing.

Urban traffic control strategies are classified as fixed-time techniques [11, 12] and model-based algorithms [13, 14]. The main drawback of the former ones is that their settings are based on historical rather than real-time data, while the latter ones basic problem is that they require algorithms with exponential complexity for a global optimization. A survey about the existing techniques is [15]. More advanced schemes have been presented recently and they refers to different models for the network and for the chosen control actions, such as max pressure control [16] and cooperative green lights policies [9]. These works consider control of intersection stages that maximizes the throughput and analyzes stability property of the network for given static demands. Conversely, the contribution of this paper regarding traffic control is the design of a strategy that optimizes in real time traffic performance and deals with continuously changing demands. Furthermore, our algorithm is formulated as a linear optimization, and it is therefore computationally very efficient.

The rest of the paper is organized as follows: Section II introduces our definition of urban network and the proposed model, including an approximation of such a model based on the average theory. Section III shows the outcomes of our validation regarding the consistency of the averaged model; Section IV discusses the choices of performance index for urban traffic while Section V describes our control strategy and presents some result of software simulations. Conclusions and future works are outlined in Section VI.

\section{SignALIZED NETWORK MODEL}

We consider urban traffic networks as sets of roads, in which the vehicles' flow passing from one road to another is regulated by traffic lights. These lights, where present, are located at the end of the roads. The crossing points, called intersections, have no capacity storage. We introduce here some preliminary notation necessary for further developments.

The roads of an urban network are a collection of three 
sets $\mathcal{R}^{\text {in }}, \mathcal{R}, \mathcal{R}^{\text {out }}$, with associated the following relations:

$$
\begin{aligned}
& \text { prev }: \mathcal{R} \cup \mathcal{R}^{\text {out }} \rightarrow \mathcal{R}^{\text {in }} \cup \mathcal{R} \\
& \text { next }: \mathcal{R}^{\text {in }} \cup \mathcal{R} \rightarrow \mathcal{R} \cup \mathcal{R}^{\text {out }},
\end{aligned}
$$

where

- $\mathcal{R}$ is the set of the inner roads of the network;

- $\mathcal{R}^{\text {in }}\left(\mathcal{R}^{\text {out }}\right)$ is the set of the roads entering (exiting) the network;

- $\operatorname{next}(r)(\operatorname{prev}(r))$ is the set of roads connected downstream (upstream) to the road $r$.

Inside the network every road in $\mathcal{R}^{\text {in }} \cup \mathcal{R}$ has at least one road downstream, while every road in $\mathcal{R} \cup \mathcal{R}^{\text {out }}$ has at least one road upstream. If $r \in \operatorname{next}(q)$ we say that $r$ and $q$ are connected to the same intersection, where $r$ is incoming and $q$ is outgoing. Note that the prev relation is undefined for the roads entering the network, while the next relation is undefined for the exiting ones.

\section{A. CTM signalized model}

To allow regulation of the traffic we introduce traffic lights as functions of the time, i.e., there exists a function

$$
\alpha: \mathbb{R}_{+} \rightarrow\{0,1\}^{\left|\mathcal{R}^{\text {in }} \cup \mathcal{R}\right|},
$$

where $\mathbb{R}_{+}$is the set of all time instants. Thus, at each time instant $t, \alpha(t)$ is a vector of $\left|\mathcal{R}^{\text {in }} \cup \mathcal{R}\right|$ values which give red (0) or green (1) to each road in $\mathcal{R}^{\text {in }} \cup \mathcal{R}$. Traffic lights operation is time-cyclic, with cycle length $T$. Typical values of $T$ are usually around 2 minutes.

To ensure the right of way (r.o.w.) we assume that:

$$
\forall q \in \mathcal{R} \cup \mathcal{R}^{\text {out }}, \quad \forall t \in \mathbb{R}_{+} \sum_{r \in \operatorname{prev}(q)} \alpha_{r}(t)=1 .
$$

We will now model the behavior of traffic flow at intersections generalizing the diverge case discussed in [3]. There is no explicit need to model the merge of flows, since they are regulated by constraint (4). In our settings each road is considered as a cell of the CTM. We further assume that associated to the network there is an array of split ratios $\beta$, where each element $\beta_{r} \in(0,1)$ represents the percentage of vehicles entered the intersection upstream to $r$ that want to go in $r$. It must be:

$$
\forall q \in \mathcal{R}^{\text {in }} \cup \mathcal{R} \quad \sum_{r \in \operatorname{next}(q)} \beta_{r}=1 .
$$

Remark 1. The values $\beta \mathrm{s}$ are supposed to be given. They may change in time, according to the network's status, but we do not consider them as variables for traffic regulation, since they are rather an indication of drivers' intentions.

We now briefly recall the demand \& supply paradigm [17], as it is necessary to describe the traffic evolution.

Definition 1. For a road $r$ we call:

- demand of $r\left(D_{r}\right)$ the flow of vehicles that want to exit $r$

- supply of $r\left(S_{r}\right)$ the flow that can be received by $r$.

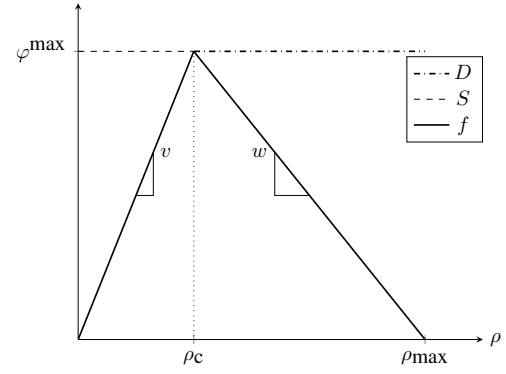

Fig. 1: Graphical representation of demand and supply functions and of the fundamental triangular diagram.

Analytical expressions for demand and supply are given through saturared functions of the density:

$$
\begin{aligned}
D_{r}\left(\rho_{r}(t)\right) & =\min \left\{v_{r} \rho_{r}(t), \varphi_{r}^{\max }\right\} \\
S_{r}\left(\rho_{r}(t)\right) & =\min \left\{\varphi_{r}^{\max }, w_{r}\left(\rho_{r}^{\max }-\rho_{r}(t)\right)\right\},
\end{aligned}
$$

where road $r$ is characterized by the following parameters: $v_{r}$, the maximum speed in freeflow; $w_{r}$, the speed in congestion phase; $\rho_{r}^{\max }$, the maximum density allowed; $\varphi_{r}^{\max }$, the maximum flow. The lowest value of density where the demand reaches its saturation value is called critical density $\left(\rho_{\mathrm{c}}\right)$.

According to the fundamental triangular diagram (see Figure 1) the relation between density and flow inside a road can be expressed as:

$$
f_{r}(t)=\min \left\{v_{r} \rho_{r}(t), w_{r}\left(\rho_{r}^{\max }-\rho_{r}(t)\right)\right\} .
$$

From the mass conservation law, the density (normalized w.r.t. the length of the road) evolves with the following rule:

$$
\dot{\rho}_{r}(t)=f_{r}^{\text {in }}(t)-f_{r}^{\text {out }}(t),
$$

where both $f_{r}^{\text {in }}$ and $f_{r}^{\text {out }}$ depend on the densities of different roads (the ones involved in the same intersection).

Consider now a road $r$ entering some intersection: the outflow of $r$ will be the maximum flow that respects the constraints given by $D_{r}$ as well as by the supply of the roads exiting the same intersection (we will introduce later the r.o.w.), i.e.,

$$
\begin{aligned}
f_{r}^{\text {out }}(t)=\max \phi & \\
\text { s.t. } \quad \phi \leq D_{r}\left(\rho_{r}(t)\right) & \\
& \beta_{j} \phi \leq S_{j}\left(\rho_{j}(t)\right) \quad \forall j \in \operatorname{next}(r) .
\end{aligned}
$$

The solution of problem (9) is given by

$$
f_{r}^{\text {out }}(t)=\min \left\{D_{r}\left(\rho_{r}(t)\right),\left\{\frac{S_{j}\left(\rho_{j}(t)\right)}{\beta_{j}}\right\}_{j \in \operatorname{next}(r)}\right\} .
$$

Thus the outflow of road $r$ will be the flow given by (10) if $r$ has r.o.w. in the downstream intersection, zero otherwise. Equation (8) becomes:

$$
\dot{\rho}_{r}(t)=f_{r}^{\text {in }}(t)-\alpha_{r}(t) f_{r}^{\text {out }}(t) .
$$

The inflow in $r$ from the upstream intersection will be:

$$
f_{r}^{\text {in }}(t)=\beta_{r} \sum_{q \in \operatorname{prev}(r)} \alpha_{q}(t) f_{q}^{\text {out }}(t) .
$$


Remark 2 (Boundary flows). Equation (10) is valid if $r \in$ $\mathcal{R}^{\text {in }} \cup \mathcal{R}$. If, instead, $r \in \mathcal{R}^{\text {out }}$ then its outflow is bounded from the supply outside the network $\left(S_{r}^{\text {out }}\right)$, i.e.,

$$
f_{r}^{\text {out }}(t)=\min \left\{D_{r}\left(\rho_{r}(t)\right), S_{r}^{\text {out }}(t)\right\} .
$$

Similarly, equation (12) is not applicable if $r \in \mathcal{R}^{\text {in }}$, where the inflow is related to the external demand $\left(D_{r}^{\text {in }}\right)$ :

$$
f_{r}^{\text {in }}(t)=\min \left\{D_{r}^{\text {in }}(t), S_{r}\left(\rho_{r}(t)\right)\right\} .
$$

Note that the model we have defined so far is consistent with the property of positive invariance [7], thanks to the demand/supply paradigm and to the constraint (4). This guarantees the consistency of the model: it will never happen that the density evolves outside the prescribed physical limits.

\section{B. Averaged model}

An interesting simplification of the model presented can be obtained applying the average theory [18, Chapter 8]. This technique looks forward for both model simplification and control strategies design. As motivations for this consider that combinatorial problems (given by the binary behavior) are usually difficult to address; Moreover, regulation of smoother variables (e.g., duty cycle) fits better to traffic scenarios, rather than fast switching between binary values. The time-average of the density (11) is:

$$
\begin{aligned}
\dot{\rho}_{r, \text { avg }} & \triangleq \frac{1}{T} \int_{t}^{t+T} \dot{\rho}_{r}(\tau) d \tau= \\
& =\frac{1}{T} \int_{t}^{t+T}\left(f_{r}^{\text {in }}(\tau)-\alpha_{r}(\tau) f_{r}^{\text {out }}(\tau)\right) d \tau .
\end{aligned}
$$

In (15) the only $T$-cyclic signal is $\alpha$, that can be averaged over the period as follows:

$$
\bar{\alpha}_{r}^{(t)} \triangleq \frac{1}{T} \int_{t}^{t+T} \alpha_{r}(\tau) d \tau=\frac{G_{r}}{T},
$$

where $G_{r}$ represents the green time of the traffic light $\alpha_{r}$. Thus $\bar{\alpha}_{r}^{(t)}$ is the duty cycle of traffic light $\alpha_{r}$ during the cycle $[t, t+T]$.

We then choose to approximate the evolution of the real density with the following:

$$
\dot{\bar{\rho}}_{r}(t)=f_{r}^{\text {in }}(t)-\bar{\alpha}_{r}^{(t)} f_{r}^{\text {out }}(t)
$$

Equation (17) represents a different dynamical system which aims to approximate (11). The flows involved in (17) are then functions of the new state variable $\bar{\rho}$. The respective of (12) will be:

$$
f_{r}^{\text {in }}(t)=\beta_{r} \sum_{q \in \operatorname{prev}(r)} \bar{\alpha}_{q}^{(t)} f_{q}^{\text {out }}(t) .
$$

In the averaged approximation the oscillating behavior of the system (due to the green/red alternation) is lost. A limitation of this approach is that possible phase shifts are not captured; a study of representation (and choice) of traffic lights' phase shifts is beyond the scope of this paper.

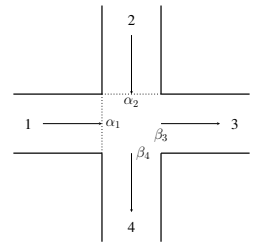

Fig. 2: Standard 4-ways intersection.

The full averaged model is given by the following set of equations:

$$
\begin{aligned}
\dot{\bar{\rho}}_{r}(t) & =f_{r}^{\text {in }}(t)-\bar{\alpha}_{r}^{(t)} f_{r}^{\text {out }}(t), r \in \mathcal{R}^{\text {in }} \cup \mathcal{R} \cup \mathcal{R}^{\text {out }} \\
f_{r}^{\text {out }}(t) & =\left\{\begin{array}{l}
\min \left\{D_{r}(t), S_{r}^{\text {out }}(t)\right\}, r \in \mathcal{R}^{\text {out }} \\
\min \left\{D_{r}(t),\left\{\frac{S_{j}(t)}{\beta_{j}}\right\}\right. \\
j \in \operatorname{next}(r)
\end{array}\right\}, \text { oth } . \\
f_{r}^{\text {in }}(t) & =\left\{\begin{array}{l}
\min \left\{D_{r}^{\text {in }}(t), S_{r}(t)\right\}, r \in \mathcal{R}^{\text {in }} \\
\beta_{r} \sum_{q \in \operatorname{prev}(r)} \bar{\alpha}_{q}^{(t)} f_{q}^{\text {out }}(t), \text { oth. }
\end{array}\right. \\
D_{r}(t) & =\min \left\{v_{r} \bar{\rho}_{r}(t), \varphi_{r}^{\max }\right\} \\
S_{r}(t) & =\min \left\{\varphi_{r}^{\max }, w_{r}\left(\rho_{r}^{\max }-\bar{\rho}_{r}(t)\right)\right\}
\end{aligned}
$$

with the constraints:

$$
\begin{aligned}
& \forall q \in \mathcal{R} \cup \mathcal{R}^{\text {out }} \quad \sum_{r \in \operatorname{prev}(q)} \bar{\alpha}_{r}^{(t)}=1 \\
& \forall q \in \mathcal{R}^{\text {in }} \cup \mathcal{R} \quad \sum_{r \in \operatorname{next}(q)} \beta_{r}=1 .
\end{aligned}
$$

Remark 3. Note that the demand and supply paradigm is fulfilled even in the averaged system: in fact, thanks respectively to (19b) and (19c), outflow and inflow for a road $r$ will satisfy the following for every $t$ :

$$
\begin{aligned}
\bar{\alpha}_{r}^{(t)} f_{r}^{\text {out }}(t) & \leq D_{r}(t) \\
f_{r}^{\text {in }}(t) & \leq \beta_{r} \sum_{q \in \operatorname{prev}(r)} \bar{\alpha}_{q}^{(t)} \frac{S_{r}(t)}{\beta_{r}}=S_{r}(t) .
\end{aligned}
$$

\section{ILLUSTRATIVE EXAMPLE AND VALIDATION}

We have built, via software simulation, a network with 40 roads connected by standard 4-ways intersections. A 4-way intersection (see Figure 2), is described by the averaged system with the following set of equations:

$$
\begin{aligned}
& \dot{\bar{\rho}}_{1}=f_{1}^{\text {in }}-\bar{\alpha}_{1} f_{1}^{\text {out }} \\
& \dot{\bar{\rho}}_{2}=f_{2}^{\text {in }}-\bar{\alpha}_{2} f_{2}^{\text {out }} \\
& \dot{\bar{\rho}}_{3}=\beta_{3}\left(\bar{\alpha}_{1} f_{1}^{\text {out }}+\bar{\alpha}_{2} f_{2}^{\text {out }}\right)-f_{3}^{\text {out }} \\
& \dot{\bar{\rho}}_{4}=\beta_{4}\left(\bar{\alpha}_{1} f_{1}^{\text {out }}+\bar{\alpha}_{2} f_{2}^{\text {out }}\right)-f_{4}^{\text {out }},
\end{aligned}
$$

where $f_{1}^{\text {in }}, f_{2}^{\text {in }}, f_{3}^{\text {out }}, f_{4}^{\text {out }}$ are the flows to be connected to other roads in the network, and constraint (4) implies $\bar{\alpha}_{1}=1-\bar{\alpha}_{2}$. For ease of visualization we assume all roads have same features: $\varphi_{r}^{\max }=\varphi^{\max }, \rho_{r}^{\max }=\rho^{\max }, v_{r}=v, w_{r}=w$, for every road $r$. We are interested in:

- Capturing the modes of the network at each time instant: a road may be either free (i.e., with density lower than the critical density) or congested (with different levels of 


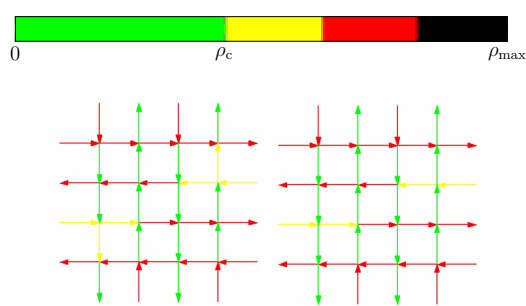

(a)

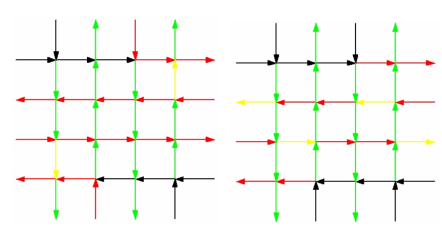

(b)

Fig. 3: Road modes at different time instants. In both $3 \mathrm{a}$ and $3 \mathrm{~b}$, on the left the actual network, on the right the averaged one, with different levels of congestion.

congestion), and we want our approximation to capture rightly as many road-modes as possible;

- Overall precision: how much the averaged system is close to the actual one, knowing that it does not present any fast switching behavior.

The simulation has been run in the following scenario: each traffic light (at each intersection) is a periodic given signal and the split ratios cause some asymmetry in the traffic diffusion. Outside of the network, we have demands (supplies) varying in time (randomly generated in $[0.5,1] \varphi^{\max }$ ) for all the entering (exiting) roads. Representative examples of our results are reported in Figures 3-4. Note that:

- The averaged system succeeds in capturing rightly an high percentage of modes. The mean error is around $10 \%$, as it results from Figure 4c. Figures $3 a$ and $3 b$ show two instantaneous pictures of the modes over the entire network (for both actual and averaged systems);

- The precision of the averaged model results to be fully satisfying in approximating the density over all roads, as shown in Figure $4 a$ and $4 b$, where the densities are very similar and the only significant difference is that oscillations in $4 \mathrm{a}$ are switched off in $4 \mathrm{~b}$, as expected.

\section{URBAN TRAFFIC PERFORMANCE METRICS}

Traffic behavior needs to be evaluated with respect to performance indices properly defined. There exist several metrics in literature to address performance evaluation; in this paper we focused on the following two features.

\section{A. Service of Demand (SoD)}

An urban traffic network is an highly dynamical environment that continously receives demand from outside. This demand cannot be ignored just to favour the inner quality of the system, because the external request will end up growing with several undesired effects, due to the bigger and bigger queues arising outside.

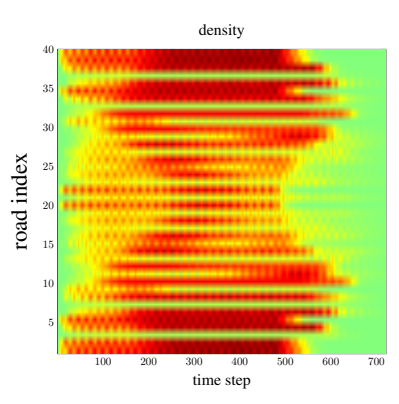

(a)

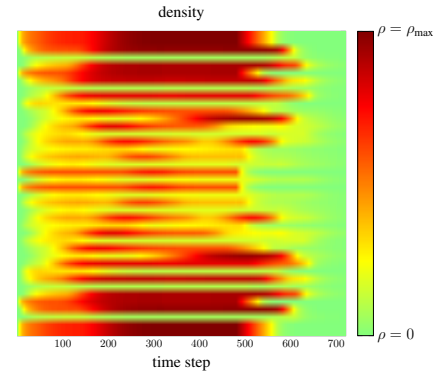

(b)

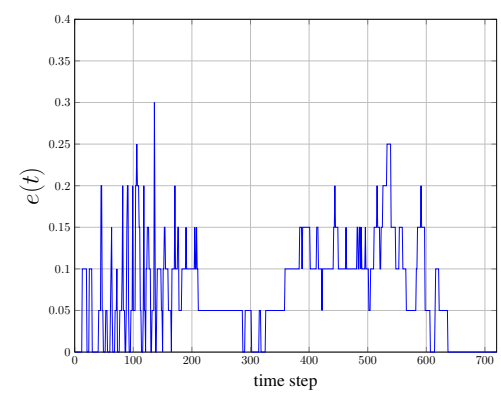

(c)

Fig. 4: In 4a (actual system) and in $4 \mathrm{~b}$ (averaged system) each row shows the density of a road evolving in time. Data showed in Fig. $4 \mathrm{c}$ is $e(t)=$ \#wrong modes $(t)$ \#roads

For this reason we define as quality of the service the number of vehicles (users) served:

$$
\begin{aligned}
\operatorname{SoD}(t) & =\int_{0}^{t} \sum_{r \in \mathcal{R}^{\text {in }}} f_{r}^{\text {in }}(\tau) d \tau= \\
& =\int_{0}^{t} \sum_{r \in \mathcal{R}^{\text {in }}} \min \left\{D_{r}^{\text {in }}(\tau), \varphi_{r}^{\max }, w_{r}\left(\rho_{r}^{\max }-\rho_{r}(\tau)\right)\right\}
\end{aligned}
$$

where $f_{r}^{\text {in }}$ is the boundary flow defined by (14) and $D_{r}^{\text {in }}$ is the external demand for road $r$. The quantity expressed in (23) is a value that we would like to maximize, since this is equivalent to decrease the sum of the queue lengths.

\section{B. Optimization of the infrastructures usage}

In urban networks some road is preferred than others by the users. The infrastructure holder would like to set traffic lights as to diminish this usage disparity, to guarantee a more equilibrate diffusion of vehicles, thus reducing hard congestions in main streets as well as the possibility of accidents.

A standard metric that takes into account this behavior is the Total Travel Distance [19], a cumulative index defined as:

$$
\operatorname{TTD}(t)=\int_{0}^{t}\left(\sum_{r \in \mathcal{R} \cup \mathcal{R}_{\text {out }}} f_{r}(\tau)\right) d \tau,
$$

where $f_{r}$ is the flow inside the road $r$, as it has been defined in (7).

TTD is a measure that should be maximized as well. 


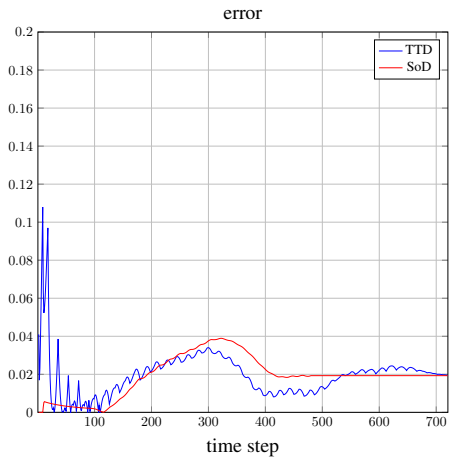

Fig. 5: Validation of the averaged model regarding the discussed metrics. The data plotted are $\frac{\left|\mathrm{SoD}(t)_{\text {actual system }}-\operatorname{SoD}(t)_{\text {averaged system }}\right|}{\operatorname{SoD}(t)_{\text {actual system }}}$, and similarly for TTD.

It is worth noting that our averaged model is able to approximate quite well even the indices functions (23) and (24). The results of these further validations are shown in Figure 5: notice that the error for both indices is lower than $10 \%$. This offers a consistent ground for the optimization of these metrics with the control strategy proposed in the next section, which is indeed based on the averaged model.

\section{PROPOSED CONTROL STRATEGY}

We propose a control algorithm for urban traffic networks based on optimization. We show that our strategy results in a linear program and, therefore, is very efficient. For ease of notation, we refer to $\bar{\alpha}^{(t)}$ as the vectors of all the $\bar{\alpha}_{r}^{(t)}$, $r \in \mathcal{R}^{\text {in }} \cup \mathcal{R}$.

At the beginning of each cycle (i.e., $t=n T, n \in \mathbb{N}$ ) we assume that a measurement of densities in the network is given. The control scheme will then decide the duty cycle $\bar{\alpha}^{(t)}$ to be applied in the upcoming cycle solving an optimization problem that involves the one-step-ahead predicted densities. We also assume that external demands and supplies are known (e.g., estimated from historical data).

\section{A. One-step-ahead optimal control}

To design the control algorithm, we first rewrite the differential equation (19a) using the Euler approximation with sampling time $\Delta t$ :

$$
\dot{\bar{\rho}}_{r} \approx \frac{\bar{\rho}_{r}(t+\Delta t)-\bar{\rho}_{r}(t)}{\Delta t},
$$

so that we can express discrete-time evolution of the density as

$$
\bar{\rho}_{r}(t+\Delta t) \approx \bar{\rho}_{r}(t)+\Delta t\left(f_{r}^{\text {in }}(t)-\bar{\alpha}_{r}^{(t)} f_{r}^{\text {out }}(t)\right) .
$$

Coherently with what said in Section IV we choose to optimize a weighted sum of indices SoD and TTD. Because of the cumulative nature of these indices, the only quantities that can be optimized one-step ahead over the duty cycles are:

$$
\begin{aligned}
\operatorname{SoD}^{+}\left(\bar{\alpha}^{(t)}\right) \triangleq \frac{1}{\Delta t}(\operatorname{SoD}(t+\Delta t)-\operatorname{SoD}(t)) \\
\operatorname{TTD}^{+}\left(\bar{\alpha}^{(t)}\right) \triangleq \frac{1}{\Delta t}(\operatorname{TTD}(t+\Delta t)-\operatorname{TTD}(t)),
\end{aligned}
$$

as at time $t$ both $\operatorname{SoD}(t)$ and $\operatorname{TTD}(t)$ are known values.

The control problem can be described with the following optimization:

$$
\begin{aligned}
\bar{\alpha}^{(t) *}= & \arg \max _{\bar{\alpha}} \sigma_{1} \operatorname{SoD}^{+}(\bar{\alpha})+\sigma_{2} \operatorname{TTD}^{+}(\bar{\alpha}) \\
& \bar{\alpha} \in \mathcal{A} \\
& \bar{\rho}_{r}(t+\Delta t)=\bar{\rho}_{r}(t)+\Delta t\left(f_{r}^{\text {in }}(t)-\bar{\alpha}_{r} f_{r}^{\text {out }}(t)\right) \\
& \text { for every } r \in \mathcal{R}^{\text {in }} \cup \mathcal{R} \cup \mathcal{R}^{\text {out }},
\end{aligned}
$$

where $f_{r}^{\text {in }}$ and $f_{r}^{\text {out }}$ are given by (19b)-(19e), while $\sigma_{1}, \sigma_{2} \geq$ 0 are weights for the involved indices.

By definition of duty cycle $\bar{\alpha}_{r}^{(t)} \in[0,1]$ for every road. These constraints, togeter with (4), give the convex domain $\mathcal{A}$ in the problem (27).

Remark 4 (Control scheme). The control first computes the values of $f_{r}^{\text {in }}(t)$ and $f_{r}^{\text {out }}(t)$, using the measured densities; then it solves the optimization problem, so computing optimal duty cycles for the upcoming cycle and finally sets them in the network. This scheme is repeated at the beginning of every cycle and not more often, in order to avoid too many switching in the traffic lights' signals.

We now show that problem (27) is truly a linear program. It can be rewritten substituting the explicit expressions of (25) and (26), and it results (we rename $\bar{\rho}_{r}^{+}(\bar{\alpha}) \triangleq \bar{\rho}_{r}(t+\Delta t)$ for sake of brevity):

$$
\begin{aligned}
\arg \max _{\bar{\alpha}}\left(\sigma_{1} \sum_{r \in \mathcal{R}^{\text {in }}} \min \left\{D_{r}^{\text {in }}(t), \varphi_{r}^{\max }, w_{r}\left(\rho_{r}^{\max }-\bar{\rho}_{r}^{+}(\bar{\alpha})\right)\right\}\right. \\
\left.+\sigma_{2} \sum_{r \in \mathcal{R} \cup \mathcal{R}^{\text {out }}} \min \left\{v_{r} \bar{\rho}_{r}^{+}(\bar{\alpha}), w_{r}\left(\rho_{r}^{\max }-\bar{\rho}_{r}^{+}(\bar{\alpha})\right)\right\}\right) \\
\bar{\alpha} \in \mathcal{A} \\
\bar{\rho}_{r}^{+}(\bar{\alpha})=\bar{\rho}_{r}(t)+\Delta t\left(f_{r}^{\text {in }}(t)-\bar{\alpha}_{r} f_{r}^{\text {out }}(t)\right),
\end{aligned}
$$

which is a convex problem. We may rewrite it as a linear optimization, doubling the number of variables, in the following way:

$$
\begin{aligned}
& \arg \max _{\bar{\alpha}, \mu, \nu}\left(\sigma_{1} \sum_{j \in \mathcal{R}^{\text {in }}} \mu_{j}+\sigma_{2} \sum_{k \in \mathcal{R} \cup \mathcal{R}^{\text {out }}} \nu_{k}\right) \\
&\left\{\begin{array}{l}
\mu_{j} \leq D_{j}^{\text {in }} \\
\mu_{j} \leq \varphi_{j}^{\text {max }} \\
\mu_{j} \leq w_{j}\left(\rho_{j}^{\max }-\bar{\rho}_{j}^{+}(\bar{\alpha})\right)
\end{array}, \forall j \in \mathcal{R}^{\text {in }}\right. \\
&\left\{\begin{array}{l}
\nu_{k} \leq v_{k} \bar{\rho}_{k}^{+}(\bar{\alpha}) \\
\nu_{k} \leq w_{k}\left(\rho_{k}^{\max }-\bar{\rho}_{k}^{+}(\bar{\alpha})\right)
\end{array}, \forall k \in \mathcal{R} \cup \mathcal{R}^{\text {out }}\right. \\
& \bar{\alpha} \in \mathcal{A} \\
& \bar{\rho}_{r}^{+}(\bar{\alpha})=\bar{\rho}_{r}(t)+\Delta t\left(f_{r}^{\text {in }}(t)-\overline{\alpha_{r}} f_{r}^{\text {out }}(t)\right) .
\end{aligned}
$$

Proposition 1. Problem (28) is equivalent to problem (29).

Proof. Notice that problem (29) is a relaxation of problem (28) and its constraints concerning variables $\mu_{j}$ and $\nu_{k}$ can be written as

$$
\begin{aligned}
& \mu_{j} \leq \min \left\{D_{j}^{\mathrm{in}}, \varphi_{j}^{\max }, w_{j}\left(\rho_{j}^{\max }-\bar{\rho}_{j}^{+}(\bar{\alpha})\right)\right\} \\
& \nu_{k} \leq \min \left\{v_{k} \bar{\rho}_{k}^{+}(\bar{\alpha}), w_{k}\left(\rho_{k}^{\max }-\bar{\rho}_{k}^{+}(\bar{\alpha})\right)\right\} .
\end{aligned}
$$




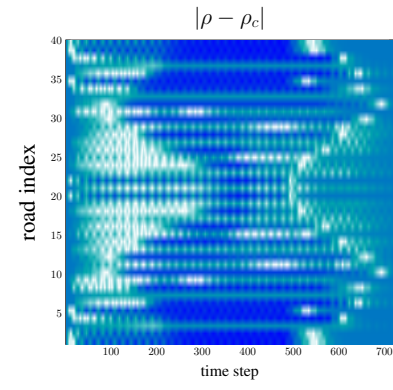

(a)

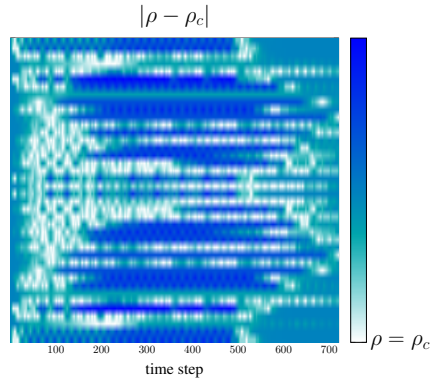

(b)
Fig. 6: Application of the proposed control strategy. Figures $6 \mathrm{a}$ and $6 \mathrm{~b}$ show the distance from the best working point $\rho_{c}$ for the network with fixed traffic lights and with our control strategy, respectively. Lighter color means better performance.

TABLE I: Improvement in the network with the proposed control strategy.

\begin{tabular}{ll}
\hline Index & Improvement $(\%)$ \\
\hline SoD & 5.5 (per entering road) \\
TTD & 14.6 \\
\hline
\end{tabular}

Hence the statement is true if at the optimum both (30) and (31) are satisfied as equality, $\forall j, k$. We then complete the proof by contradiction. Suppose $\mu^{*}, \nu^{*}$ is optimal and there exist a real positive number $\varepsilon$ and an integer $i$ such that

$$
\mu_{i}^{*}+\varepsilon=\min \left\{D_{i}^{\text {in }}, \varphi_{i}^{\max }, w_{i}\left(\rho_{i}^{\max }-\bar{\rho}_{i}^{+}(\bar{\alpha})\right)\right\}
$$

(the same reasoning applies if the variable in point is some $\left.\nu_{i}^{*}\right)$. Then we may add $\varepsilon$ to the variable $\mu_{i}^{*}$ and we will obtain a new admissible solution (note that in (29) $\mu_{i}$ is uncorrelated with $\mu_{j}, i \neq j$ ). Because of the maximization purpose, such a new solution will be better than the previous, which, therefore, could not be optimal.

\section{B. Simulation and results}

To test the proposed control algorithm we have run simulations using the software [20] to solve the optimization problem. We stress again the fact that the control is actuated over the non-averaged system. The test has been run for a virtual time of one hour, during which demands are varying and they are randomly generated in $[0.5,1] \varphi^{\max }$, in order to test the control in different circumstances. The metrics SoD and TTD are evaluated over the whole simulation horizon and compared with the ones obtained with a fixed-light policy based only on the split ratios (and not on real-time optimization). Representative results are shown in Figure 6 and in Table I. Note that:

- Our algorithm achieves good performance regarding the optimization of the infrastructures usage. Figure 6 shows how far each road's density is from its best working point $\rho_{c}$, where lighter color means better performance;

- Table I gives the quantitative measures of the improvement, which is positive for both the choosen indices.

\section{CONCLUSIONS AND FUTURE WORKS}

In this paper we presented a model for urban signalized traffic networks and its average-based approximation for control purposes. Numerical validations have confirmed the reliability of such approximation. We also proposed a numerically efficient control scheme based on global optimization, whose application in simulation has given good performance improvements.

Future research will aim to design multi-step-ahead control, combined with techniques for traffic lights phase shift.

\section{REFERENCES}

[1] M. J. Lighthill and G. B. Whitham. "On kinematic waves. II." In: Proceedings of the Royal Society of London. Series A. 229 (1955), pp. 317-345.

[2] C. F. Daganzo. "The cell transmission model: A dynamic representation of highway traffic consistent with the hydrodynamic theory", In: Transportation Research Part B: Methodological 28.4 (1994), pp. 269-287.

[3] C. F. Daganzo. "The cell transmission model, part II: network traffic". In: Transportation Research Part B: Methodological 29.2 (1995), pp. 79-93.

[4] C. F. Daganzo and N. Geroliminis. "An analytical approximation for the macroscopic fundamental diagram of urban traffic". In: Transportation Research Part B: Methodological 42.9 (2008), pp. 771-781.

[5] G. Como, K. Savla, D. Acemoglu, M. A Dahleh, and E. Frazzoli. "Robust distributed routing in dynamical networks-part I: Locally responsive policies and weak resilience". In: Automatic Control, IEEE Transactions on 58.2 (2013), pp. 317-332.

[6] G. Como, K. Savla, D. Acemoglu, M. A Dahleh, and E. Frazzoli. "Robust distributed routing in dynamical networks-part II: Strong resilience, equilibrium selection and cascaded failures". In: Automatic Control, IEEE Transactions on 58.2 (2013), pp. 333-348.

[7] S. Coogan and M. Arcak. "Dynamical Properties of a Compartmental Model for Traffic Networks". In: Proceedings of the 52nd IEEE American Control Conference (2014), pp. 5951-5956.

[8] E. Lovisari, G. Como, A. Rantzer, and Savla K. "Stability analysis and control synthesis for dynamical transportatio networks". Under review, available at arXiv:1410.5956v2.

[9] K. Savla, E. Lovisari, and G. Como. "On Maximally Stabilizing Traffic Signal Control with Unknown Turn Ratios". In: World Congress 19.1 (2014), pp. 1849-1854.

[10] K. Aboudolas, M. Papageorgiou, and E. Kosmatopoulos. "Store-andforward based methods for the signal control problem in large-scale congested urban road networks". In: Transportation Research Part C: Emerging Technologies 17.2 (2009), pp. 163-174.

[11] J.D.C. Little. "The synchronization of traffic sisgnals by mixedinteger-linear-programming". In: Oper. Res. 14 (1966), pp. 568-594.

[12] D.I. Robertson. "TRANSYT method for area traffic control". In: Traffic Eng. Control 10 (1969), pp. 276-281.

[13] J.L. Farges, J.J. Henry, and J. Tufal. "The PRODYN real-time traffic algorithm”. In: Proc. 4th IFAC SYmp. Transportation Systems (1983), pp. 307-312.

[14] S. Sen and L. Head. "Controlled optimization of phases at an intersection". In: Transp. Sci. 31 (1997), pp. 5-17.

[15] M. Papageorgiou, C. Diakaki, V. Dinopoulou, A. Kotsialos, and Y. Wang. "Review of road traffic control strategies". In: Proceedings of the IEEE 91.12 (2003), pp. 2043-2067.

[16] P. Varaiya. "Max pressure control of a network of signalized intersections". In: Transportation Research Part C: Emerging Technologies 36 (2013), pp. 177-195.

[17] J.P. Lebacque. "The Godunov scheme and what it means for firstorder traffic models". In: Proceedings of the 13th International Symposium on Transportation and Traffic Theory (1996), pp. 647-677.

[18] H.K. Khalil. Nonlinear systems. Prentice Hall, 2002.

[19] G. Gomes and R. Horowitz. "Optimal freeway ramp metering using the asymmetric cell transmission model". In: Transportation Research Part C: Emerging Technologies 14.4 (2006), pp. 244-262.

[20] J. Löfberg. "YALMIP : A Toolbox for Modeling and Optimization in MATLAB". In: Proceedings of the CACSD Conference. Taipei, Taiwan, 2004. 